\title{
Diferencias de género en el trastorno delirante.
}

Gender differences in delusional disorder.

\author{
Nuria Román Avezuela a, Nuria Esteve Díaz beticia Domarco Manrique c, Alberto Domínguez

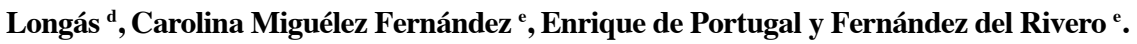 \\ a,b,c, d, e yf Psiquiatra. ${ }^{a}$ Unidad de Salud Mental. Hospital Universitario de Torrejón. Torrejón de Ardoz. \\ Madrid, España. ${ }^{b}$ Clínica Neuropsiquiátrica Dr. León. Madrid, España. ${ }^{c}$ Clinique Marigny. Toulousse, \\ Francia ${ }^{d}$ Centro de Salud Mental Villa de Vallecas. Madrid, España. ${ }^{e}$ Departamento de Psiquiatría. Hos- \\ pital General Universitario Gregorio Marañón. Madrid, España.
}

Correspondencia: Nuria Román Avezuela (nuriaroman@hotmail.es)

Recibido: 29/08/2014; aceptado: 01/09/2014

RESUMEN: El objetivo de nuestro estudio fue explorar las diferencias de género en trastorno delirante (TD) comparando aspectos sociodemográficos, factores de riesgo, correlatos clínicos y características del curso del trastorno. La muestra se compuso de 50 pacientes con primer ingreso psiquiátrico y con diagnóstico de TD (según SCID-I para criterios DSM-IV). Los casos fueron evaluados retrospectivamente mediante la revisión de historia clínicas mediante el cuestionario OPCRIT $4.0 \mathrm{y}$ un inventario diseñado por los autores que comprendió distintos aspectos clínicos no recogidos en el anterior instrumento. La proporción mujer-hombre fue de 1.27:1. La edad del primer ingreso psiquiátrico fue más alta en mujeres. Respecto a los potenciales factores de riesgo, los hombres presentaron mayor prevalencia de Trastornos por uso de sustancias premórbido mientras las mujeres más retraimiento social y síntomas depresivos previos al inicio del trastorno. En relación a la sintomatología delirante, los hombres presentaron mayor frecuencia de delirios generalizados, de grandiosidad, de celos y persecución; mientras que las mujeres presentaron mayor frecuencia de delirio erotomaniaco así como percepciones y humor delirantes. Los hombres presentaron mayor frecuencia de aumento de la estima del Self y las mujeres insomnio inicial y de media noche. No se observaron diferencias de género en otros factores sociodemográficos, potenciales factores de riesgo, correlatos clínicos y características del curso de la enfermedad.

PALABRAS CLAVE: Género, diferencias, trastorno delirante, psicosis, psicopatología, factores de riesgo.
ABSTRACT: The objective of our study was to explore gender differences in Delusional Disorder (DD) comparing sociodemographic, clinical and risk factors and course's characteristics. The sample was compound of 50 inpatients during their first psychiatric entrance and diagnosis of DD (according to SCID-I for DSM-IV criteria). Cases were evaluated retrospectively by means of medical records revision, OPCRIT 4.0 and an inventory created by authors, about not gathered aspects. The proportion woman-man was of 1.27:1. The age of the first psychiatric entrance was higher in women. Men presented greater prevalence of Disorders for use of drugs before; while the women suffered more social retirement and previous depressive symptoms. Men presented greater frequency of generalized, megalomaniac, and jealousy and persecution delusions; whereas women displayed greater frequency of erotomaniac delusions, as well as delusional affectivity and perceptions. Men presented greater frequency of increase of the esteem of the Self; and women displayed initial insomnia and of average night more frequently. Differences in other sociodemographic, clinical and risk factors, and course's characteristics were not observed.

KEY WORDS: Gender, differences, delusional disorder, psychosis, psychopathology, risk factors. por su esfuerzo, rigor, dedicación y tesón, sin los que hubiera sido imposible llevar a cabo el presente estudio. 
ORIGINALES Y REVISIONES

\section{Introducción}

Aunque en las dos últimas décadas el interés por el estudio del trastorno delirante (TD) ha aumentado, éste sigue siendo una de las enfermedades mentales menos estudiadas y más desconocidas (1-4). El TD se incluyó como entidad nosológica independiente en el DSM-III en 1987 por hallarse en estudios previos dos diferencias relevantes respecto a la esquizofrenia paranoide (EP) y al trastorno depresivo mayor (TDM): (i) la edad inicio del TD es significativamente mayor que en la esquizofrenia y (ii) la frecuencia de mujeres en el TD es significativamente más baja que la de los trastornos afectivos (5). Hasta donde conocemos, sólo se han publicado cuatro estudios con solidez metodológica considerable en los que el objetivo principal fue explorar las diferencias de género existentes en el TD (3-4, 6-7).

\subsection{Género, factores demográficos y de riesgo.}

La mayoría de los estudios previos han demostrado que la enfermedad es más prevalente en mujeres que en hombres (2, 5, 8-10). Algunos estudios determinan que los hombres se encuentran con mayor frecuencia solteros, mientras que las mujeres se encuentran casadas $(9,11)$ y viudas $(2)$. Respecto a los potenciales factores de riesgo para el TD, hay varios estudios que han hallado que los varones presentan mayores antecedentes de consumo de sustancias y de traumatismos craneoencefálicos (3, 11-12). En las mujeres parece que tendría mayor relevancia la presencia de factores psicosociales precipitantes y los antecedentes familiares de psicosis (2).

1.2. Correlatos clínicos y psicopatológicos de género.

El prototipo masculino de Paranoia / TD descrito por Rudden y cols. en 1983 consiste en la presencia de ideación delirante de contenido homosexual, mientras que el prototipo femenino se caracteriza por la presencia de ideas delirantes erotomaniacas heterosexuales (6). Según de Portugal y cols. (2010), el TD celotípico (según DSM-IV) es más frecuente en hombres, lo que es consistente con las descripciones que realizó Enoch $(2,13)$. Existe una sólida que demuestra que las mujeres presentan con mayor frecuencia comorbilidad de trastornos depresivos y mayor severidad de éstos (6-9).

\subsection{Género, curso y funcionalidad}

Se ha observado que los varones tardan menos tiempo en consultar con un psiquiatra desde el inicio TD $(5,10)$, y que las mujeres presentan una edad de inicio más tardío $(11,14)$. También se ha detectado que las mujeres con TD presentan una peor funcionamiento premórbido y que la tasa de remisión del trastorno es menor (4).

1.4. Objetivos y justificación del estudio

Dado que los estudios anteriormente descritos se basaron en muestras con una marcada heterogeneidad clínica, presentaron hallazgos contradictorios y no existe ningún estudio previo disponible hasta la fecha, que específicamente explore las di- 
ORIGINALES Y REVISIONES

ferencias de género en una muestra de TD durante el primer ingreso psiquiátrico, se nos ofrece una oportunidad excepcional para comparar las diferencias de género existentes en: (1) los aspectos sociodemográficos; (2) los potenciales factores de riesgo; (3) los correlatos clínicos; (4) el curso y funcionamiento social en TD durante el comienzo de la enfermedad.

\section{Método}

\subsection{Diseño}

Estudio retrospectivo de un Registro de Casos.

\subsection{Muestra}

Se marcó como objetivo en la selección de casos reclutar los 50 primeros ingresos psiquiátricos de pacientes con diagnostico de TD. El Hospital General Universitario Gregorio Marañón (HGUGM) es un hospital perteneciente a la red pública de la Consejería de Sanidad de la Comunidad de Madrid que asume la atención psiquiátrica directa de unos 750.000 ciudadanos en una zona bien definida del sureste de Madrid. Para alcanzar el tamaño de la muestra propuesto en nuestro estudio, se realizaron los siguientes pasos: 1) Se obtuvo un primer registro de casos a través del sistema informático de información específico del HGUGM (SISCAM) de todos los pacientes diagnosticados de TD (DSM-III R y DSM-IV) / Trastorno por ideas delirantes persistentes (CIE-10) (TIDP) que ingresaron en el HGUGM desde el año 2000 hasta el 2010; 2) De este primer registro de casos, se seleccionaron de manera consecutiva los 50 primeros casos de TD que ingresaron a partir del año 2000 hasta el 2010 y que cumplieron los siguientes criterios de inclusión y exclusión. Los criterios de inclusión fueron: 1) Confirmación del diagnostico de TD / TIDP mediante revisión del diagnóstico emitido en el informe de alta del paciente a través de la Historia Clínica Informatizada (HCI) y/o Historia Clínica en Papel (HCP); 2) Primer ingreso psiquiátrico en el HGUGM mediante revisión de la HCI o HCP; y 3) Edad del paciente mayor de 18 años. Mientras que los criterios de exclusión fueron: 1) Historia de un ingreso previo en cualquier hospital con diagnóstico de TD/ TIDP o EP; 2) Dependencia o abuso de drogas directamente relacionado con la aparición del TD; 3) Retraso mental; 4) Historia actual o pasada de enfermedad cerebral orgánica relevante; 5) No cumplir la confirmación diagnostica de TD según OPCRIT (Operational Criteria Checklist for Psychotic Illness and Affective Illness) para DSM-IV (15).

\subsection{Variables e Instrumentos.}

Todos los pacientes fueron evaluados mediante la revisión de HCI y/o HCP por un médico especialista en formación de psiquiatría entrenado para utilizar todos los instrumentos del estudio. El diagnóstico de TD se confirmo mediante el OPCRIT 
ORIGINALES Y REVISIONES

4.0, que es un instrumento conveniente, validado y ampliamente utilizado, del que se ha establecido su fiabilidad (15). Además, se empleó un inventario sistematizado (IS) que fue diseñado por los autores para explorar aquellas variables clínicas que no fueron recogidas a través del OPCRIT: 1) variables sociodemográficas: edad del primer ingreso psiquiátrico con diagnóstico de TD, raza, relación de pareja, tipo de convivencia, desempeño de una actividad formativa o laboral, nivel educacional alcanzado; 2) potenciales factores de riesgo: consumo activo de sustancias un mes antes del inicio del trastorno, abuso/dependencia a alcohol (DSM-IV), abuso/dependencia a cannabis (DSM-IV), déficit auditivo premórbido, inmigración al inicio del trastorno, barrera idiomática al inicio del trastorno, estresores al inicio del trastorno, marcado aislamiento social al inicio del trastorno, síntomas depresivos previos al inicio del trastorno, síntomas depresivos presentes al inicio del trastorno, trastornos psiquiátricos concomitantes, patología médica concomitante; 3) ideas y creencias delirantes: delirio de referencia, de persecución, somático, de Schneider, erotomaniaco y de celos. 4) alucinaciones: auditivas no prominentes, visuales, gustativas $y / u$ olfatorias.

\subsubsection{Inventario sistematizado elaborado por los autores (IS)}

I. Factores sociodemográficos:

1. Edad en el momento de su primer ingreso psiquiátrico con diagnóstico de TD/TIDP (años)

2. Raza caucásica (afirmativo/negativo)

3. Mantenía una relación de pareja estable en el momento de su primer ingreso psiquiátrico con diagnóstico de TD/TIDP (afirmativo/negativo)

4. Sobre el tipo de convivencia, vive solo en el momento de su primer ingreso psiquiátrico con diagnóstico de TD/TIDP (afirmativo/negativo)

5. Se encontraba desarrollando una actividad formativa o profesional en el momento de su primer ingreso psiquiátrico con diagnóstico de TD/TIDP (afirmativo/negativo)

6. Disponía de un nivel educacional de graduado escolar o inferior en el momento de su primer ingreso psiquiátrico con diagnóstico de TD/TIDP (afirmativo/negativo)

7. Tiempo de escolarización en el momento de su primer ingreso psiquiátrico con diagnóstico de TD/TIDP (años) 
II. Potenciales factores de riesgo:

1. Consumo sustancias 1 mes antes del inicio del trastorno con patrón de consumo perjudicial, abuso o dependencia (afirmativo/negativo)

2. Presentaba antecedentes de déficit auditivo premórbido al inicio del trastorno (afirmativo/negativo)

3. Se encontraba en situación de inmigración al inicio del trastorno (afirmativo/negativo)

4. Se encontraba con una barrera idiomática respecto a la mayoría de la población al inicio del trastorno (afirmativo/negativo)

5. Presencia de factores estresores psicosociales significativos al inicio del trastorno (afirmativo/negativo)

6. Situación social era de marcado aislamiento durante el inicio del trastorno (afirmativo/negativo)

7. Presentaba antecedentes de T. Depresivo anterior al inicio del trastorno (afirmativo/negativo)

8. Presencia de síntomas depresivos significativos al inicio de la aparición de las primeras ideas delirantes (afirmativo/negativo)

9. Presencia de otro Trastorno Psiquiátrico concomitante al inicio del trastorno (afirmativo/negativo)

10. Presencia de patología médica concomitante al inicio del trastorno (afirmativo/negativo)

III. Correlatos clínicos y psicopatológicos

1. Presencia de ideas delirantes de referencia durante su primer ingreso (afirmativo/negativo)

2. Presencia de ideas delirantes somáticas durante su primer ingreso (afirmativo/negativo)

3. Presencia de ideas delirantes de Schneider durante su primer ingreso (afirmativo/negativo)

4. Presencia de ideas delirantes erotomaniacas durante su primer ingreso (afirmativo/negativo)

5. Presencia de ideas delirantes de celos durante su primer ingreso (afirmativo/negativo)

6. Presencia de alucinaciones auditivas no prominentes durante su primer ingreso (afirmativo/negativo)

7. Presencia de alucinaciones visuales durante su primer ingreso (afirmativo/negativo)

8. Presencia de alucinaciones tactiles durante su primer ingreso (afirmativo/ negativo)

9. Presencia de alucinaciones gustativas y/o olfativas durante su primer ingreso (afirmativo/negativo) 
ORIGINALES Y REVISIONES

\subsection{Análisis estadísticos}

La prueba $t$ de Student y el análisis de varianza (ANOVA) se utilizaron para las variables continuas con distribución normal. Las pruebas no paramétricas (test de U de Mann-Whitney y de Kruskal Wallis) se aplicarán para las variables continuas que no cumplan una distribución normal. La prueba Chi-cuadrado y la prueba exacta Fisher se utilizarán para las comparaciones de variables cualitativas.

\section{Resultados}

\subsection{Género y factores sociodemográficos.}

Las características sociodemográficas se muestran en la tabla 1.

Respecto a los factores sociodemográficos, las mujeres representan el $56 \%(\mathrm{n}=28)$ de la muestra y los varones, el 44\% ( $\mathrm{n}=22)$. Las mujeres con diagnóstico de TD ingresan por primera vez en psiquiatría a edades más tardías (52.07 y 45 años respectivamente) con tendencia a la significación estadística. No se detectan diferencias estadísticamente significativas en factores sociodemográficos como son estado civil, raza caucásica, tipo de convivencia, desempeño de actividad formativa y/u ocupacional, nivel educacional o número de años estudiados.

\subsection{Género y factores de riesgo.}

Los resultados acerca de los potenciales factores de riesgo se muestran en la tabla 1.

Hemos detectado que los hombres abusan más frecuentemente de sustancias que las mujeres ( $40.9 \%$ y $3.6 \%$ respectivamente), tienen una mayor prevalencia de diagnóstico de abuso o dependencia de cannabis (22.7\% y $0 \%$, respectivamente) y de alcohol (22.7\% y $3.6 \%$, respectivamente), antecedentes de consumo activo de sustancias un mes antes del inicio del trastorno ( $40.9 \%$ y $3.6 \%$, respectivamente) de forma significativa. La presencia de aislamiento social marcado $(67.9 \%$ y $38.1 \%$, respectivamente) y de síntomas depresivos previos al inicio del trastorno $(35.7 \%$ y $9.1 \%$, respectivamente) son más frecuentes en mujeres que en hombres de forma significativa.

No se han detectado diferencias de género estadísticamente significativas en otros potenciales factores de riesgo. 
Tabla 1.

Diferencias de género en los factores y potenciales factores de riesgo en trastorno delirante.

\begin{tabular}{|c|c|c|c|c|}
\hline \multicolumn{5}{|c|}{ Trastorno delirante $(\mathrm{n}=\mathbf{5 0})$} \\
\hline & $\begin{array}{c}\text { Mujeres } \\
n=28\end{array}$ & $\begin{array}{c}\text { Varones } \\
n=22\end{array}$ & $\begin{array}{c}\text { Estadístico } \\
a / b\end{array}$ & $P$ \\
\hline \multicolumn{5}{|l|}{ Factores sociodemográficos } \\
\hline Edad, primer ingreso psiquiátrico (IS) & $\begin{array}{c}52.07 \\
D S=16.07\end{array}$ & $\begin{array}{c}45.00 \\
\mathrm{DS}=12.04\end{array}$ & $1.718^{\mathrm{b}}$ & $\overline{0.092}$ \\
\hline Estado civil, soltero (O-6) & $10(35.7 \%)$ & $10(45.5 \%)$ & $1.182^{\mathrm{a}}$ & 0.757 \\
\hline Raza caucásica (IS) & $2796.4 \%)$ & $22(100 \%)$ & $0.000^{\mathrm{a}}$ & $>0.999$ \\
\hline Relación de pareja estable (IS) & $6(21,4 \%)$ & $7(31,8 \%)$ & $0.691^{\mathrm{a}}$ & 0.460 \\
\hline Tipo de convivencia, vive solo (IS) & $14(50,0 \%)$ & $8(36,4 \%)$ & $0.930^{\mathrm{a}}$ & 0.335 \\
\hline Actividad formativa o profesional (IS) & $13(46.4 \%)$ & $10(45,5 \%)$ & $0.005^{\mathrm{a}}$ & 0.945 \\
\hline Graduado escolar o sin estudios (IS) & $15(62,5 \%)$ & $10(62.5 \%)$ & - & - \\
\hline Tiempo escolarizado (años) (IS) & $\begin{array}{c}12.48 \\
\mathrm{DS}=4.24 \\
\end{array}$ & $\begin{array}{c}12.73 \\
\mathrm{DS}=3.35 \\
\end{array}$ & $-0.170^{\mathrm{b}}$ & 0.866 \\
\hline \multicolumn{5}{|l|}{ Potenciales factores de riesgo } \\
\hline Historia familiar esquizofrenia $(\mathrm{O}-13)$ & $1(3.6 \%)$ & 0 & $0.000^{\mathrm{a}}$ & $>0.999$ \\
\hline Historia familiar otro T. Mental (O-14) & $4(14.3 \%)$ & $4(18.2 \%)$ & $0.139^{\mathrm{a}}$ & 0.709 \\
\hline T. Personalidad premórbido (O-11) & $7(25 \%)$ & $9(40.9 \%)$ & $1.433^{\mathrm{a}}$ & 0.231 \\
\hline Abuso sustancias premórbido (O-12) & $1(3.6 \%)$ & $9(40.9 \%)$ & $10.735^{\mathrm{a}}$ & $0.001 * *$ \\
\hline Consumo sustancias 1 mes antes (IS) & $1(3.6 \%)$ & $9(40.9 \%)$ & $10.735^{\mathrm{a}}$ & $0.001 * *$ \\
\hline Abuso o dependencia alcohol (O-78) & $1(3.6 \%)$ & $5(22.7 \%)$ & $4.083^{\mathrm{a}}$ & 0.043* \\
\hline Abuso o dependencia cannabis $(\mathrm{O}-79)$ & 0 & $5(22.7 \%)$ & $4.771^{\mathrm{a}}$ & 0.029* \\
\hline Trastorno cerebral previo (O-15) & $2(7.1 \%)$ & $1(4.5 \%)$ & $0.000^{\mathrm{a}}$ & $>0.999$ \\
\hline Claro estresor psicosocial (O-16) & $12(42.9 \%)$ & $10(45.5 \%)$ & $0.034^{\mathrm{a}}$ & 0.854 \\
\hline Déficit auditivo premórbido (IS) & $1(3.6 \%)$ & 0 & $0.000^{\mathrm{a}}$ & $>0.999$ \\
\hline Inmigrante al inicio del trastorno (IS) & $2(7.1 \%)$ & 0 & $0.305^{\mathrm{a}}$ & 0.581 \\
\hline Barrera idiomática al inicio (IS) & $1(3.6 \%)$ & 0 & $0.000^{\mathrm{a}}$ & $>0.999$ \\
\hline Estresores al inicio del trastorno (IS) & $12(42.9 \%)$ & $7(31.8 \%)$ & $0.637^{\mathrm{a}}$ & 0.425 \\
\hline Marcado aislamiento social (IS) & $19(67.9 \%)$ & $8(38.1 \%)$ & $4.296^{\mathrm{a}}$ & 0.038* \\
\hline T. Depresivo previo al trastorno (IS) & $10(35.7 \%)$ & $2(9.1 \%)$ & $4.788^{\mathrm{a}}$ & $0.029^{*}$ \\
\hline T. Depresivo al inicio del trastorno (IS) & $9(32.1 \%)$ & $6(27.3 \%)$ & $0.139^{a}$ & 0.709 \\
\hline T. Psiquiátrico concomitante (IS) & $9(32.1 \%)$ & $9(40.9 \%)$ & $0.411^{\mathrm{a}}$ & 0.522 \\
\hline Patología médica concomitante (IS) & $8(28.6 \%)$ & $8(36.4 \%)$ & $0.344^{\mathrm{a}}$ & 0.558 \\
\hline
\end{tabular}

(†) $\mathrm{p}<0.1 ;(*) \mathrm{p}<0.05 ;(* *) \mathrm{p}<0.01$.

(a) Chi cuadrado; (b) T Student.

Abreviaciones: O (OPCRIT), Operational Checklist for Psychotic Symptoms. IS, Inventario sistematizado. 
3.2. Género, ideación delirante, otros trastornos del pensamiento y alteraciones de la percepción.

Las características clínicas y psicopatológicas referentes a ideación delirante, otros trastornos del pensamiento y alteraciones de la percepción se exponen en la tabla 2.

Tabla 2 .

Diferencias de género en ideación delirante, otros trastornos del pensamiento y alteraciones de la percepción en trastorno delirante.

\begin{tabular}{|c|c|c|c|c|}
\hline \multicolumn{5}{|c|}{ Trastorno delirante $(\mathrm{n}=\mathbf{5 0})$} \\
\hline & $\begin{array}{c}\text { Mujeres } \\
\mathrm{n}=28\end{array}$ & $\begin{array}{c}\text { Varones } \\
\mathrm{n}=22 \\
\end{array}$ & $\begin{array}{c}\text { Estadístico } \\
a / b \\
\end{array}$ & $\bar{P}$ \\
\hline \multicolumn{5}{|l|}{ Ideas y creencias anormales } \\
\hline Delirio de persecución (O-54) & $26(92.9 \%)$ & $19(86.4 \%)$ & $0.577^{\mathrm{a}}$ & 0.447 \\
\hline Delirio bien organizado (O-55) & $28(100 \%)$ & $22(100 \%)$ & - & - \\
\hline Aumento estima del Self (O-56) & $1(3.6 \%)$ & $6(27.3 \%)$ & $3.948^{\mathrm{a}}$ & $0.047^{*}$ \\
\hline Delirio de grandiosidad (O-57) & 0 & $4(18.2 \%)$ & $3.339^{\mathrm{a}}$ & $0.068^{\dagger}$ \\
\hline Delirio de influencia (O-58) & 0 & 0 & - & - \\
\hline Delirio bizarro (O-59) & $1(3.6 \%)$ & $0 \%$ & $0.000^{\mathrm{a}}$ & $>0.999$ \\
\hline Delirio generalizado (O-60) & $20(71.4 \%)$ & $20(90.6 \%)$ & $2.922^{\mathrm{a}}$ & $0.087^{\dagger}$ \\
\hline Delirio de pasividad (O-61) & $1(3.6 \%)$ & 0 & $0.000^{\mathrm{a}}$ & $>0.999$ \\
\hline Percepción delirante $1^{\mathrm{a}}(\mathrm{O}-62)$ & $11(39.3 \%)$ & $2(9.1 \%)$ & $5.838^{\mathrm{a}}$ & $0.016^{*}$ \\
\hline Humor delirante (O-63) & $9(32.1 \%)$ & $2(9.1 \%)$ & $3.815^{\mathrm{a}}$ & $\mathbf{0 . 0 5 1}{ }^{\dagger}$ \\
\hline Delirios y/o alucinaciones (O-64) & $27(96.4 \%)$ & $22(100 \%)$ & $0.000^{\mathrm{a}}$ & $>0.999$ \\
\hline Delirio persecución y celos (O-65) & $18(64.3 \%)$ & $19(86.4 \%)$ & $3.121^{\mathrm{a}}$ & $\mathbf{0 . 0 7 7 ^ { \dagger }}$ \\
\hline Pensamiento inducido (O-66) & 0 & 0 & - & - \\
\hline Robo del pensamiento (O-67) & 0 & 0 & - & - \\
\hline Difusión del pensamiento (O-68) & $1(3.6 \%)$ & 0 & $0.000^{\mathrm{a}}$ & $>0.999$ \\
\hline Eco del pensamiento (O-72) & 0 & 0 & - & - \\
\hline Delirio de culpa (O-69) & $1(3.6 \%)$ & 0 & $0.000^{\mathrm{a}}$ & $>0.999$ \\
\hline Delirio de pobreza (O-70) & 0 & 0 & - & - \\
\hline Delirio nihilistico (O-71) & 0 & $1(4.5 \%)$ & $0.015^{\mathrm{a}}$ & 0.903 \\
\hline Delirio de referencia (IS) & $23(82.1 \%)$ & $18(81.8 \%)$ & $0.001^{\mathrm{a}}$ & 0.976 \\
\hline Delirio somático (IS) & $2(7.1 \%)$ & $5(22.7 \%)$ & $1.358^{\mathrm{a}}$ & 0.244 \\
\hline Delirio de Schneider (IS) & $1(3.6 \%)$ & 0 & $0.000^{\mathrm{a}}$ & $>0.999$ \\
\hline Delirio erotomaniaco (IS) & $6(21.4 \%)$ & 0 & $3.520^{\mathrm{a}}$ & $0.061^{\dagger}$ \\
\hline Delirio de celos (IS) & $1(3.6 \%)$ & $5(22.7 \%)$ & $2.659^{\mathrm{a}}$ & 0.103 \\
\hline \multicolumn{5}{|l|}{ Pensamiento y habla } \\
\hline Dificultad entendimiento (O-26) & $2(7.1 \%)$ & 0 & $0.305^{\mathrm{a}}$ & 0.581 \\
\hline Habla incoherente $(\mathrm{O}-27)$ & $1(3.6 \%)$ & $1(4.5 \%)$ & $0.000^{\mathrm{a}}$ & $>0.999$ \\
\hline T. Positivo formal (O-28) & $1(3.6 \%)$ & 0 & $0.000^{\mathrm{a}}$ & $>0.999$ \\
\hline T. Negativo formal (O-29) & 0 & 0 & - & - \\
\hline Habla apresurada (O-30) & $5(17.9 \%)$ & $3(13.9 \%)$ & $0.000^{\mathrm{a}}$ & 0.988 \\
\hline Pensamiento rápido (O-31) & $1(3.6 \%)$ & 0 & $0.000^{\mathrm{a}}$ & $>0.999$ \\
\hline \multicolumn{5}{|l|}{ Alucinaciones } \\
\hline Auditivas en $3^{\mathrm{a}}$ persona $(\mathrm{O}-73)$ & 0 & 0 & - & - \\
\hline Voces que comentan (O-74) & 0 & 0 & - & - \\
\hline Voces persecutorias (O-75) & $3(10.7 \%)$ & 0 & $0.968^{\mathrm{a}}$ & 0.325 \\
\hline Auditivas no afectivas (O-76) & $4(14.3 \%)$ & $1(4.5 \%)$ & $0.442^{\mathrm{a}}$ & 0.506 \\
\hline Auditivas no prominentes (IS) & $6(21.4 \%)$ & $1(4.5 \%)$ & $1.683^{\mathrm{a}}$ & 0.195 \\
\hline Visuales (IS) & $1(3.6 \%)$ & 0 & $0.000^{\mathrm{a}}$ & $>0.999$ \\
\hline Táctiles (IS) & $2(7.1 \%)$ & 0 & $0.305^{\mathrm{a}}$ & 0.581 \\
\hline Gustativas y/u olfativas (IS) & $1(3.6 \%)$ & $1(4.5 \%)$ & $0.000^{\mathrm{a}}$ & $>0.999$ \\
\hline
\end{tabular}

(i) $\mathrm{p}<0.1 ;(*) \mathrm{p}<0.05 ;(* *) \mathrm{p}<0.01$.

(a) Chi cuadrado; (b) T Student.

Abreviaciones: O (OPCRIT), Operational Checklist for Psychotic Symptoms. IS, Inventario sistematizado. 
Los varones presentaron más frecuentemente aumento de la autoestima del Self (27.3\% y $3.6 \%$, respectivamente) de forma significativa; y delirios generalizados ( $90.6 \%$ y $71.4 \%$, respectivamente), ideas delirantes de grandiosidad (18.2\% y $0 \%$, respectivamente) y delirios de persecución y celos (86.4\% y $64.3 \%$, respectivamente) con tendencia a la significación estadística.

En las mujeres se observó más frecuentemente percepción delirante primaria (39.3\% y 9.1\%, respectivamente) de forma significativa; y delirios erotomaniacos ( $21,4 \%$ y $0 \%$, respectivamente) y humor delirante ( $32.1 \%$ y $9.1 \%$, respectivamente) con tendencia a la significación.

No se han observado otras diferencias de género en las ideas y creencias delirantes, formas de pensamiento, habla y alucinaciones.

\subsection{Género, afecto y ritmos biológicos.}

Los hallazgos clínicos y psicopatológicos referentes a la esfera afectiva y los ritmos biológicos se exponen en la tabla 3 .

Tabla 3 .

Diferencias de género en el afecto y ritmos biológicos en trastorno delirante.

\begin{tabular}{|c|c|c|c|c|}
\hline \multicolumn{5}{|c|}{ Trastorno delirante $(\mathrm{n}=\mathbf{5 0})$} \\
\hline & $\begin{array}{c}\text { Mujeres } \\
\mathrm{n}=28\end{array}$ & $\begin{array}{c}\text { Varones } \\
n=22\end{array}$ & $\begin{array}{c}\text { Estadístico } \\
a / b\end{array}$ & $\boldsymbol{P}$ \\
\hline \multicolumn{5}{|l|}{ Esfera afectiva } \\
\hline Afecto restringido (O-32) & $1(3.6 \%)$ & $1(4.5 \%)$ & $0.000^{\mathrm{a}}$ & $>.999$ \\
\hline Afecto embotado (O-33) & 0 & 0 & - & - \\
\hline Afecto inapropiado (O-34) & $1(3.6 \%)$ & $1(4.5 \%)$ & $0.000^{\mathrm{a}}$ & $>.999$ \\
\hline Ánimo elevado (O-35) & $1(3.6 \%)$ & $1(4.5 \%)$ & $0.000^{\mathrm{a}}$ & $>.999$ \\
\hline Ánimo irritable (O-36) & $12(42.9 \%)$ & $8(36.4 \%)$ & $0.216^{\mathrm{a}}$ & 0.642 \\
\hline Disforia $(\mathrm{O}-37)$ & $10(35.7 \%)$ & $9(40.9 \%)$ & $0.141^{\mathrm{a}}$ & 0.707 \\
\hline Variación diurna $(\mathrm{O}-38)$ & 0 & 0 & - & - \\
\hline Pérdida de placer (O-39) & $3(17.6 \%)$ & $3(15 \%)$ & $0.000^{\mathrm{a}}$ & $>.999$ \\
\hline Disminución líbido (O-40) & $1(6.3 \%)$ & 0 & $0.004^{\mathrm{a}}$ & 0.952 \\
\hline Pobre concentración (O-41) & $2(7.1 \%)$ & $2(9.5 \%)$ & $0.000^{\mathrm{a}}$ & $>.999$ \\
\hline Excesivo reproche Self $(\mathrm{O}-42)$ & $1(3.6 \%)$ & 0 & $0.000^{\mathrm{a}}$ & $>.999$ \\
\hline Ideación suicida (O-43) & $3(10.7 \%)$ & $3(13.6 \%)$ & $0.000^{\mathrm{a}}$ & $>.999$ \\
\hline Relación psicótico-afecto (O-52) & $17(60.7 \%)$ & $10(45.5 \%)$ & $1.155^{\mathrm{a}}$ & 0.283 \\
\hline \multicolumn{5}{|l|}{ Ritmos biológicos } \\
\hline Insomnio inicial (O-44) & $16(61.5 \%)$ & $17(31.8 \%)$ & $4.218^{\mathrm{a}}$ & $0.040 *$ \\
\hline Insomnio media noche $(\mathrm{O}-45)$ & $10(43.5 \%)$ & $4(19.0 \%)$ & $3.020^{\mathrm{a}}$ & $\mathbf{0 . 0 8 2}$ \\
\hline Despertar temprano (O-46) & $4(15.4 \%)$ & $1(4.5 \%)$ & $0.564^{\mathrm{a}}$ & 0.453 \\
\hline Sueño excesivo $(\mathrm{O}-47)$ & 0 & 0 & - & - \\
\hline Escaso apetito (O-48) & $5(19.2 \%)$ & $3(13.6 \%)$ & $0.017^{\mathrm{a}}$ & 0.897 \\
\hline Pérdida de peso (O-49) & $3(12.0 \%)$ & $3(13.6 \%)$ & $0.000^{\mathrm{a}}$ & $>.999$ \\
\hline Aumento de apetito (O-50) & 0 & 0 & - & - \\
\hline Aumento de peso (O-51) & 0 & 0 & 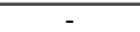 & - \\
\hline
\end{tabular}

(†) $\mathrm{p}<0.1 ;(*) \mathrm{p}<0.05 ;(* *) \mathrm{p}<0.01$.

(a) Chi cuadrado; (b) T Student.

Abreviaciones: O (OPCRIT), Operational Checklist for Psychotic Symptoms. IS, Inventario sistematizado. 
Las mujeres padecen más a menudo insomnio inicial $(61.5 \%$ y $31.8 \%$, respectivamente) de forma significativa, e insomnio de media noche $(43.5 \%$ y $19 \%$, respectivamente) con tendencia a la significación.

No se han observado otras diferencias de género en la esfera afectiva y en los ritmos biológicos.

3.4. Género, conducta y otros aspectos.

Las características clínicas y psicopatológicas referentes a las alteraciones conductuales, en la capacidad de insight y en la detección de información no creíble se muestran en la tabla 4.

Tabla 4.

Diferencias de género en la conducta y otros aspectos en trastorno delirante.

\begin{tabular}{|c|c|c|c|c|}
\hline \multicolumn{5}{|c|}{ Trastorno delirante $(n=50)$} \\
\hline & $\begin{array}{c}\text { Mujeres } \\
\mathrm{n}=28\end{array}$ & $\begin{array}{c}\text { Varones } \\
n=22\end{array}$ & $\begin{array}{c}\text { Estadístico } \\
a / b\end{array}$ & $\boldsymbol{P}$ \\
\hline \multicolumn{5}{|l|}{ Conducta } \\
\hline Comportamiento extraño (O-17) & $12(42.9 \%)$ & $6(27.3 \%)$ & $1.299^{\mathrm{a}}$ & 0.254 \\
\hline Catatonía (O-18) & 0 & 0 & - & - \\
\hline Actividad excesiva (O-19) & $1(3.6 \%)$ & $3(13.6 \%)$ & $0.604^{\mathrm{a}}$ & 0.437 \\
\hline Conducta imprudente (O-20) & $2(7.1 \%)$ & $4(18.2 \%)$ & $0.568^{\mathrm{a}}$ & 0.451 \\
\hline Distractibilidad (O-21) & $1(3.6 \%)$ & 0 & $0.000^{\mathrm{a}}$ & $>.999$ \\
\hline Reducida necesidad sueño (O-22) & 0 & $1(4.5 \%)$ & $0.011^{\mathrm{a}}$ & 0.917 \\
\hline Actitud nerviosa (O-23) & $17(60.7 \%)$ & $14(63.6 \%)$ & $0.045^{\mathrm{a}}$ & 0.833 \\
\hline Poca actividad (O-24) & $1(3.6 \%)$ & 0 & $0.000^{\mathrm{a}}$ & $>.999$ \\
\hline Pérdida de energía (O-25) & $1(3.6 \%)$ & $5(22.7 \%)$ & $2.659^{\mathrm{a}}$ & 0.103 \\
\hline Aumento sociabilidad (O-53) & $1(3.6 \%)$ & 0 & $0.000^{\mathrm{a}}$ & $>.999$ \\
\hline \multicolumn{5}{|l|}{ Otros } \\
\hline Información no creíble (O-84) & 0 & 0 & - & - \\
\hline Ausencia de insight (O-85) & $28(100 \%)$ & $21(95.5 \%)$ & $1.299^{\mathrm{a}}$ & 0.254 \\
\hline
\end{tabular}

$\left({ }^{\dagger}\right) \mathrm{p}<0.1 ;(*) \mathrm{p}<0.05 ;(* *) \mathrm{p}<0.01$.

(a) Chi cuadrado; (b) T Student.

Abreviaciones: O (OPCRIT), Operational Checklist for Psychotic Symptoms. IS, Inventario sistematizado.

No se han observado diferencias de género en las alteraciones conductuales, en la capacidad de insight y en la detección de información no creíble.

3.3. Género, curso, funcionalidad y evolución.

Las diferencias de género en el curso, funcionalidad y evolución del TD se exponen en la tabla 5. lidad.

No se encontraron otras diferencias de género en curso, evolución o funciona- 
Tabla 5.

Diferencias de género en curso, evolución y funcionalidad en trastorno delirante.

\begin{tabular}{|c|c|c|c|c|}
\hline \multicolumn{5}{|c|}{ Trastorno delirante $(n=50)$} \\
\hline & $\begin{array}{c}\text { Mujeres } \\
n=28\end{array}$ & $\begin{array}{c}\text { Varones } \\
n=22\end{array}$ & $\begin{array}{c}\text { Estadístico } \\
a / b\end{array}$ & $p$ \\
\hline $1^{\text {a }}$ consulta a psiquiatría (años) $(\mathrm{O}-4)$ & $\begin{array}{c}46.78 \\
\mathrm{DS}=17.77 \\
\end{array}$ & $\begin{array}{c}41.85 \\
\mathrm{DS}=9.72 \\
\end{array}$ & $1.120^{\mathrm{b}}$ & 0.269 \\
\hline $\begin{array}{l}\text { Duración de la enfermedad (semanas) } \\
(\mathrm{O}-8)\end{array}$ & $\begin{array}{c}89.29 \\
\mathrm{DS}=25.25 \\
\end{array}$ & $\begin{array}{c}77.76 \\
\mathrm{DS}=37.10 \\
\end{array}$ & $1.294^{\mathrm{b}}$ & 0.202 \\
\hline Desempleado antes del inicio (O-7) & $16(59.3 \%)$ & $12(63.2 \%)$ & $0.071^{\mathrm{a}}$ & 0.790 \\
\hline Pobre adaptación al trabajo (O-9) & $8(28.6 \%)$ & $7(35.0 \%)$ & $0.224^{\mathrm{a}}$ & 0.636 \\
\hline Pobre adaptación premórbida social (O-10) & $6(21.4 \%)$ & $7(35 \%)$ & $1.088^{\mathrm{a}}$ & 0.297 \\
\hline Deterioro funcional premórbido (O-88) & $9(32.1 \%)$ & $4(18.2 \%)$ & $1.248^{\mathrm{a}}$ & 0.264 \\
\hline Respuesta a antipsicóticos (O-89) & $20(71.4 \%)$ & $18(81.8 \%)$ & $0.729^{\mathrm{a}}$ & 0.393 \\
\hline Buena recuperación $(\mathrm{O}-90)$ & $7(25,9 \%)$ & $7(31,8 \%)$ & $0.206^{\mathrm{a}}$ & 0.650 \\
\hline
\end{tabular}

(†) $\mathrm{p}<0.1 ;(*) \mathrm{p}<0.05 ;(* *) \mathrm{p}<0.01 . b h$

(a) Chi cuadrado; (b) T Student.

Abreviaciones: O (OPCRIT), Operational Checklist for Psychotic Symptoms. IS, Inventario sistematizado.

\section{Discusión}

\subsection{Limitaciones y fortalezas.}

Nuestros resultados tienen limitaciones a considerar que dificultan su generalización. Primero, la muestra no es homogénea respecto a la distribución de género (28 mujeres y 22 hombres), pero esto posiblemente refleje una mayor prevalencia en mujeres. Segundo, el tamaño muestral es reducido $(n=50)$, por tanto, puede no haberse detectado algunas diferencias entre géneros porque la potencia del estudio no sea suficiente. Tercero, se trata de un estudio retrospectivo de un registro de casos en el que se realiza una recogida sistematizada de datos clínicos tras revisión de HCP y/o HCI empleando un inventario diseñado por los autores (Anexo) y OPCRIT (15), por lo que debemos de interpretar con precaución.

Una de las fortalezas de nuestro estudio es que en la recogida sistematizada de datos se ha empleado una entrevista estructurada (OPCRIT) cuya validez ha sido demostrada mediante estudios sólidos (15). Otra fortaleza a señalar es que se toma una muestra de pacientes durante su primer ingreso psiquiátrico con diagnóstico de TD, lo cual resulta un diseño idóneo para el análisis de factores de riesgo y clínicos sin la influencia de la cronicidad del trastorno.

Por tanto, los resultados obtenidos en nuestro estudio sólo se deberían extrapolar a pacientes con TD de corta evolución y en fase de descompensación psicopatológica en un área específica. 
ORIGINALES Y REVISIONES

\subsection{Género, factores demográficos y de riesgo.}

Uno de los hallazgos observados a señalar, es que el trastorno delirante es más frecuente en mujeres que en hombres en un ratio de 1.27:1 (28 mujeres y 22 hombres). Otros estudios han observado esta mayor proporción del TD en mujeres $(3,5$, 7-10). Sin embargo, dicho hallazgo no es consistente con DSM-IV, ni con el estudio publicado recientemente por Wustmann y cols. (2011), en los cuales se concluye que no existen diferencias en la prevalencia de TD entre hombres y mujeres $(4,16)$. Se ha objetivado que las mujeres con diagnóstico de TD ingresan por primera vez en psiquiatría a edades más tardías (52.07 y 45 años respectivamente), congruentemente con otros tres estudios publicados $(4,11,14)$. No se detectan diferencias significativas en el estado civil, al igual que en otro estudio publicado recientemente (4), y a diferencia de otros estudios, en los que los varones se encontraban más frecuentemente solteros y las mujeres, viudas o casadas $(3,9,11)$.

Con respecto a los potenciales factores de riesgo, los hombres presentan más frecuentemente diagnóstico de abuso de sustancias premórbido, diagnóstico de abuso o dependencia de alcohol y de cannabis y antecedentes de uso de sustancias con patrón de consumo perjudicial, de abuso o dependencia un mes antes del inicio del trastorno, lo cual es congruente con varios estudios previos (3,11-12). Las mujeres presentan de forma significativa más frecuentemente aislamiento social marcado y síntomas depresivos previos al inicio del trastorno, este hallazgo concuerda con los resultados obtenidos en varios estudios previos $(4,6-8,17)$ y con Rudden y cols. cuando afirman que el TD en mujeres es más parecido al trastorno esquizoafectivo que en el hombre (6). La existencia por una parte de una prevalencia de trastornos por uso de sustancias (TUS) mayor en varones, y por otra, de más síntomas depresivos y aislamiento social en mujeres, es congruente con varios estudios que han observado que el TD en hombres posee mayor sustrato orgánico, mientras que en mujeres los factores precipitantes interpersonales juegan un papel más importante en el desarrollo del trastorno $(3,6,11,14)$. No hemos encontrado diferencias de género en otros potenciales factores riesgo como antecedentes de traumatismo cerebral previo, al contrario que otros estudios previos donde se observó que eran más frecuentes en varones $(3,11-12)$. No se han observado diferencias en la presencia de antecedentes familiares de psicosis, aunque en un estudio previo ha observado que son más frecuentes en mujeres (2). Tampoco se han hallado diferencias de género en la presencia de barrera idiomática, inmigración, diagnóstico de trastorno de personalidad previo, déficit sensorial, síntomas depresivos presentes al inicio del trastorno, trastornos psiquiátricos o patología orgánica concomitante al igual que de Portugal y cols. (3).

4.2. Correlatos clínicos y psicopatológicos de género.

Hemos objetivado que los varones presentan más frecuentemente aumento de la estima del Self de forma significativa, e ideas delirantes de grandiosidad con tendencia a la significación. Estos hallazgos son congruentes con que en las mujeres se 
ORIGINALES Y REVISIONES

objetive más frecuentemente sintomatología depresiva (3, 6, 17-18). Ninguno de los estudios previos disponibles hasta la fecha han observado que las ideas delirantes de tipo grandioso o el aumento de la estima del Self presenten diferencias de género TD $(2-3,7,10,18)$. Así mismo, los varones presentaron más a menudo delirios de persecución y celos con tendencia a la significación. Los trabajos publicados por Rudden y cols. y de Portugal y cols. también han determinado que el subtipo de celos es más frecuente en hombres, tal y como describía Enoch en su libro "Síndrome de Othello" $(3,6,13)$. La presencia de delirios generalizados ha sido con tendencia significativa mayor en hombres que en mujeres. En las mujeres se observó más frecuentemente humor delirante con tendencia a la significación; y percepción delirante primaria e ideación erotomaniaca de forma significativa. Rudden y cols. en su trabajo publicado en 1983, también observó que los delirios erotomaniacos eran más frecuentes en mujeres (6). Las mujeres padecen más a menudo insomnio inicial de forma significativa e insomnio de media noche con tendencia a la significación. Ningún estudio previo ha explorado diferencias de género en la presencia de delirios generalizados, percepción delirante primaria, humor delirante, insomnio de conciliación o mixto (1-3, 5, 7-8, 14, 17-19).

No se detectan diferencias en la presencia de alucinaciones auditivas, incongruentemente con algún estudio previo en el que se objetiva que los varones presentan más alucinaciones auditivas (3). No se han encontrado diferencias en cuanto a síntomas negativos a diferencia de otros trabajos $(3,5,14)$, lo cual, posiblemente, pueda relacionarse con que nuestro estudio evalúa una muestra de pacientes en su primer ingreso hospitalario en el que reciben el diagnóstico al alta de TD, por lo que el tiempo de evolución del trastorno es menor que en otros estudios y la prevalencia de síntomas negativos sea insuficiente para detectar diferencias entre sexos.

\subsection{Género, curso y funcionalidad}

No se detectan diferencias de género en relación a la edad a la que se consulta por primera vez a un psiquiatra, al igual que en un estudio de Bada y cols. (7); sin embargo, Kendler y cols. en su trabajo publicado en 1982, objetivaron que los varones consultan antes (5). No fueron halladas diferencias en la duración total de la enfermedad a diferencia del estudio de Wustmann y cols. (2001), en el que se objetiva que el diagnóstico de TD es más estable y crónico en mujeres (4). No se encontraron diferencias de género significativas en factores pronósticos relacionados con la situación psicosocial previa al desarrollo de la enfermedad, sin embargo, en otro estudio anterior se observó que las mujeres presentaban peor ajuste psicosocial previo (4). No se objetivaron diferencias en la respuesta a tratamiento neuroléptico entre hombres y mujeres, ni en la remisión sintomática al alta hospitalaria, coincidiendo con los resultados obtenidos por Bada y cols., (7). Estos datos contradicen los resultados del trabajo de Wustmann y cols., en el que se observó que las mujeres presentan mayor tendencia a la cronicidad del cuadro aunque el cumplimiento terapéutico en ellas sea 
ORIGINALES Y REVISIONES

mejor (4). A diferencia del estudio de Wustmann y cols., nuestro estudio toma una muestra de pacientes con TD durante su primer ingreso, posiblemente el tiempo de evolución sea insuficiente para detectar diferencias entre géneros en este sentido.

\section{Conclusiones}

El presente trabajo es un estudio retrospectivo de un registro de casos, en el que hemos comparado las diferencias de género existentes en factores sociodemográficos, clínicos y características del curso del trastorno en una muestra de 50 pacientes durante su primer ingreso psiquiátrico con diagnóstico de TD empleando un inventario sistematizado diseñado por los autores (Anexo) y OPCRIT 4.0 (15).

Sobre los factores sociodemográficos, se ha podido objetivar un predominio de mujeres $(1,27: 1)$ y que éstas presentan una edad en su primer ingreso más elevada.

Atendiendo a los factores de riesgo, los varones presentaron con mayor frecuencia antecedentes de abuso de sustancias premórbido, diagnóstico de abuso/dependencia a cannabis y alcohol premórbidos y uso de sustancias un mes antes con patrón de consumo perjudicial, de abuso o dependencia. Las mujeres presentaron mayor frecuencia de aislamiento social marcado y sintomatología depresiva previa al inicio del trastorno.

En lo referente a la clínica y la psicopatología, los hombres presentaron más frecuentemente aumento de la estima del Self, delirios grandiosos, de perjuicio y celos, y generalizados. Entre las mujeres se detectó una mayor frecuencia de delirios erotomaniacos, percepción y humor delirantes, insomnio inicial y de media noche.

No se detectaron otras diferencias de género en factores sociodemográficos, de riesgo, clínicos o características del curso del trastorno.

Nuestros resultados tienen limitaciones a considerar que dificultan su generalización. Se trata de un estudio retrospectivo de un registro de pacientes diagnosticados de TD en su primer ingreso psiquiátrico, con un reducido tamaño muestral $(n=50)$ y distribución entre géneros no homogénea por lo que recomendamos que los hallazgos encontrados se interpreten con precaución.

\section{BIBLIOGRAFÍA}

(1) de Portugal E, Cervilla JA. Trastorno delirante desde un punto de vista empírico. Monografías de psiquiatría $2007 \mathrm{Sept}$;19(3):3-18.

(2) de Portugal E, Gonzalez N, Haro JM, Autonell J, Cervilla JA. A descriptive case-register study of delusional disorder. Eur Psychiatry 2008 Mar;23(2):125-33. 
(3) de Portugal E, Gonzalez N, Miriam V, Haro JM, Usall J, Cervilla JA. Gender differences in delusional disorder: Evidence from an outpatient sample. Psychiatry Res 2010 May 15;177(12):235-9.

(4) Wustmann T, Pillmann F, Marneros A. Gender-related features of persistent delusional disorders. Eur Arch Psychiatry Clin Neurosci 2011;261:29-36.

(5) Kendler KS. Demography of paranoid psychosis (delusional disorder): a review and comparison with schizophrenia and affective illness. Arch Gen Psychiatry 1982 Aug;39(8):890-902.

(6) Rudden M, Sweeney J, Frances A, Gilmore M. A comparison of delusional disorders in women and men. Am J Psychiatry 1983 Dec;140(12):1575-8.

(7) Bada A, Albert U, Maina G, Bogetto F. Gender effects on clinical features in delusional disorder. Italian Journal of Psicopathology 2000;6:4.

(8) Maina G, Albert U, Bada A, Bogetto F. Occurrence and clinical correlates of psychiatric co-morbidity in delusional disorder. Eur Psychiatry 2001 Jun;16(4):222-8.

(9) Marino C, Nobile M, Bellodi L, Smeraldi E. Delusional disorder and mood disorder: can they coexist? Psychopathology 1993;26(2):53-61.

(10) Yamada N, Nakajima S, Noguchi T. Age at onset of delusional disorder is dependent on the delusional theme. Acta Psychiatr Scand 1998 Feb;97(2):122-4.

(11) Munro A, Mok H. An overview of treatment in paranoia/delusional disorder. Can J Psychiatry 1995 Dec;40(10):616-22.

(12) Galderisi S, Bucci P, Ucok A, Peuskens J. No gender differences in social outcome in patients suffering from schizophrenia. Eur Psychiatry 2011 May 24.

(13) Enoch D, Ball H. The Othello syndrome. In: Enoch D, Ball H, editors. Uncommon psychiatric syndromes. 4th ed. London: Arnold; 2001. p. 50-73.

(14) Munro A. Phenomenological aspects of monodelusional disorders. Br J Psychiatry Suppl 1991 Nov(14):62-4.

(15) Rucker J, Newman S, Gray J, Gunasinghe C, Broadbent M, Brittain P, et al. OPCRIT+: an electronic system for psychiatric diagnosis and data collection in clinical and research settings. $\mathrm{Br}$ J Psychiatry 2011 Aug;199:151-5.

(16) APA. Diagnostic and statistical manual of mental disorders (DSM-IV). 4th ed. Washington, DC1994.

(17) Grover S, Biswas P, Avasthi A. Delusional disorder: Study from North India. Psychiatry Clin Neurosci2007 Oct;61(5):462-70.

(18) Hsiao MC, Liu CY, Yang YY, Yeh EK. Delusional disorder: retrospective analysis of 86 Chinese outpatients. Psychiatry Clin Neurosci 1999 Dec;53(6):673-6.

(19) Serretti A, Lattuada E, Cusin C, Smeraldi E. Factor analysis of delusional disorder symptomatology. Compr Psychiatry 1999 Mar-Apr;40(2):143-7. 